\title{
Time-of-day-dependent Effects of Monochromatic Light Exposure on Human Cognitive Function
}

\author{
Ming $\mathrm{An}^{1)}$, Jinghua Huang ${ }^{2)}$, Yoshihiro Shimomura ${ }^{2)}$ and Tetsuo Katsuura ${ }^{2)}$ \\ 1) Humanomics Section, Graduate School of Science and Technology, Chiba University \\ 2) Humanomics Section, Graduate School of Engineering, Chiba University
}

\begin{abstract}
Light elicits non-visual effects on a wide range of biological functions and behavior. These effects are mediated by a melanopsin-based photoreceptor system that is very sensitive to blue light $(440-480 \mathrm{~nm})$ relative to the three-cone visual photopic system. The aim of the current study was to assess the time-of-day-dependent effects of two different wavelength monochromatic lights at $458 \mathrm{~nm}$ and $550 \mathrm{~nm}$ on human cognitive function. We conducted an experiment in the daytime and nighttime on different days. Twelve subjects were selected, none of whom was either morning-type or eveningtype, as assessed by a translated version of the morningness/ eveningness questionnaire. The cognitive function was measured by event-related potential (ERP) using an oddball task, and arousal level was measured by the Alpha Attenuation Test (AAT). We found that $458 \mathrm{~nm}$ light exposure caused a significantly larger P300 amplitude than occurred with $550 \mathrm{~nm}$ light. There was a significant interaction among wavelength, time of day, and electrode site. Exposure to $458 \mathrm{~nm}$ light induced a larger P300 amplitude at nighttime than in the daytime at the Fz electrode site. The Alpha Attenuation Coefficient (AAC) at nighttime was higher than in the daytime. Our results suggest that short wavelength monochromatic light can affect the circadian rhythms of cognitive functions, and indicate that these effects are mediated by a melanopsin-based photoreceptor system. This study has extended previous findings in terms of time of day, and higher cognitive function by using an endogenous ERP component, P300. J Physiol Anthropol 28(5): 217-223, 2009 http://www.jstage.jst.go.jp/ browse/jpa2
\end{abstract}

[DOI: 10.2114/jpa2.28.217]

Keywords: circadian rhythm, light wavelength, cognitive function, auditory event-related potential, P300

\section{Introduction}

Since ancient times, human adaptability to the environment, including the lighting environment, has been one of the main subjects of physiological anthropology (Sato, 2005), so functional analyses of the time-of-day effects of monochromatic light exposure on human cognition have become one of the important themes in this field.

It is well known that the rod and cone photopigments are involved in visual responses. Since Berson et al. (2002) detected a novel photoreceptor cell, melanopsin, an intrinsic photosensitive retinal ganglion cell (ipRGC) in the retina of mammals, which is most sensitive to short-wavelength visible light, it began to emerge that the eye performs a dual role in detecting light for a range of behavioral and physiological responses that are distinct from the classical visual responses. Melanopsin-containing ipRGCs have their own nerve connections to a range of 'non-visual' areas of the brain, including the suprachiasmatic nucleus ( $\mathrm{SCN}$ ), which is the biological clock of the brain. The SCN has a nerve connection with the pineal gland, which is responsible for the regulation of some types of hormones, as well as with many areas that share input from the visual photoreceptor system, such as the lateral geniculate nucleus, pretectum, and superior colliculus.

In humans, ocular light exposure plays a crucial role in circadian-phase resetting (Boivin et al., 1996; Wever et al., 1983; Zeitzer et al., 2000; Zaidi et al., 2007; Lockley et al., 2003; Warman et al., 2003; Deacon and Arendt, 1995). Light also elicits acute physiological effects in humans such as a rapid suppression of the nocturnal pineal hormone melatonin (Lewy et al., 1980; Bojkowski et al., 1987; Brainard et al., 1997) and increases in core body temperature (CBT) (Badia et al., 1991; Cajochen et al., 1992; Cajochen et al., 2000; Dijk et al., 1991) and heart rate (Scheer et al., 2004; Cajochen et al., 2005). Light also induces pupillary constriction (Zaidi et al., 2007). Many studies have been devoted to studying the effects of bright light on human objective alertness with the electroencephalogram (EEG) or functional magnetic resonance imaging (fMRI) during the nighttime hours (Lockley et al., 2006; Zaidi et al., 2007; Myers and Badia, 1993; Badia et al., 1991; Campbell and Dawson, 1990), and daytime hours (Vandewalle et al., 2007). For example, Lockley et al. (2006), who measured light-induced changes in EEG correlates of 
arousal, have shown that $460 \mathrm{~nm}$ monochromatic light is more effective than $555 \mathrm{~nm}$ light at suppressing delta/theta activity $(0.5-5 \mathrm{~Hz})$, which is considered a marker of both the circadian and homeostatic drive for arousal; furthermore, blue light preferentially activates high-alpha frequency oscillations $(9.5-10.5 \mathrm{~Hz})$. In addition, Zaidi et al. (2007) showed that blue light preferentially increased alpha activity $(8-10 \mathrm{~Hz})$ in EEG recordings, indicating a more alert state. Only a few studies (Katsuura et al., 2007) have been devoted to studying the effect of monochromatic light on human cognitive function with ERP. However, they evaluated central nervous activity using P300 event-related potentials under red and blue light exposure at the same illuminance level (310 lx at the level of the eye). We know that non-visual effects have action spectra that differ from those of vision; thus, measures of light for vision using photopic weighting of the incident spectrum (i.e., using a luxmeter) are not suitable for the evaluation of any non-visual effect. We should control the irradiance when assessing the spectral characteristics of light. ERP is a sequence of electrical changes elicited by sensory or perceptual stimuli, and cognitive events. P300 is a component of the ERP that was discovered by Sutton et al. (1965) and is elicited when subjects attend to a discrimination task. This procedure has been dubbed the 'oddball paradigm,' since two stimuli are presented in a random series such that one of them, i.e., the oddball, occurs relatively infrequently. The subject is required to distinguish the stimuli by noticing the occurrence of every target (mental counting, button pressing) and by not responding to the standard stimulus. The target stimulus elicits P300, which is not apparent in the ERP from the standard stimulus. P300 is a general measure of "cognitive efficiency" in the sense of how well and how rapidly a person's central nervous system can process and incorporate new information (Polich, 1998). In particular, P300 amplitude indexes brain actions stemming from 'tasks that are required in the maintenance of working memory' (Donchin et al., 1986) when the mental model or context of the stimulus environment is updated (Donchin, 1981; Donchin and Coles, 1988). P300 latency is considered to be a sensitive measure of stimulusclassification speed (Polich et al., 1983) and is generally unrelated to response selection and execution processes (Pfefferbaum et al., 1986; Kok, 1997). Thus, P300 can be considered to be a manifestation of central nervous system activity involved in the processing of new information when attention is engaged to update memory representations (Verleger, 1988; Polich and Kok, 1995; Polich, 1996; Kok, 1997; Coull, 1998).

Kleitman (1939) noticed that alertness showed a circadian rhythm, with its maximum in the evening and nadir in the early morning, about 70 years ago. Several studies showed that human cognitive function exhibits diurnal variation indexed by ERP (Broughton et al., 1988; Wesensten et al., 1990; Higuchi et al., 2000; Huang et al., 2006) and suggested that the measurement of P300 allows the objective identification of circadian rhythm in cognitive function (Higuchi et al., 2000;
Huang et al., 2006). However, to our knowledge, no study has explored the time-of-day effects of human cognitive function during monochromatic light exposure indexed by P300.

In the present study, we aimed to demonstrate the time-ofday-dependent effects of two different wavelengths of light, $458 \mathrm{~nm}$ and $550 \mathrm{~nm}$, on human higher cognitive function by using the endogenous ERP component, P300.

\section{Methods}

\section{Subjects and prestudy conditions}

A total of 12 male healthy undergraduate students (mean age $=20.92, \mathrm{SD}=1.08 \mathrm{yrs}$ ) participated in the present study. In order to minimize inter-individual variation in circadian phase, only subjects who were neither evening nor morning types (average scores $=49.67 \pm 4.65$, average waking time $=7: 52 \pm$ $1: 16$, average bedtime $=24: 27 \pm 0: 35 \mathrm{hr}$ ) were selected, as assessed by a translated version of the morningness/ eveningness questionnaire (Horne and Östberg, 1976). This questionnaire consisted of 19 questions pertaining to habitual bed and waking times, preferred times of physical and mental performance, and subjective fatigue after rising and before going to bed. The questionnaire yields scores on a single scale of 'morningness' vs 'eveningness' ranging from 16 to 86 . Higher scores suggest greater 'morningness.' Lower scores indicate greater 'eveningness.' Horne and Östberg (1976) classify subjects who score $70-86$ on their questionnaire as 'definitely morning type,' those who score 59-69 as 'moderately morning type,' those who score $42-58$ as 'neither type,' those who score $31-41$ as 'moderately evening type,' and those who score 16-30 as 'definitely evening type.' They reported normal hearing and no neurological or psychological problems. All subjects had normal color vision according to the Farnsworth-Munsell 100 Hue Test. The subjects were instructed to keep a regular sleep-wake schedule during the week (baseline days) before entering the study in order to synchronize their sleep-wake rhythm, and kept the same schedule until the completion of this study. Compliance with the protocol was confirmed by sleep diaries and actigraphic recordings (Actiwatch-L, Minimitter, Inc., Bend, OR). Subjects were asked to abstain from the use of any prescription or recreational drugs, alcohol, and caffeine or nicotine. Subjects gave written informed consent and were paid for their participation.

\section{Study protocol}

The present experiments were performed two times (daytime and nighttime), $12 \mathrm{~h}$ out of phase with each other. Both protocols consisted of the same set of measurements but differed in terms of the timings of light exposure. The protocols are summarized in Fig. 1. Daytime and nighttime experiments took place on 2 separate days, a protocol that required at least 3 days of interval to avoid learning effects, and were assigned to subjects in a counterbalanced manner. In each session, after $5 \mathrm{~min}$ of standard light exposure (19 lx 


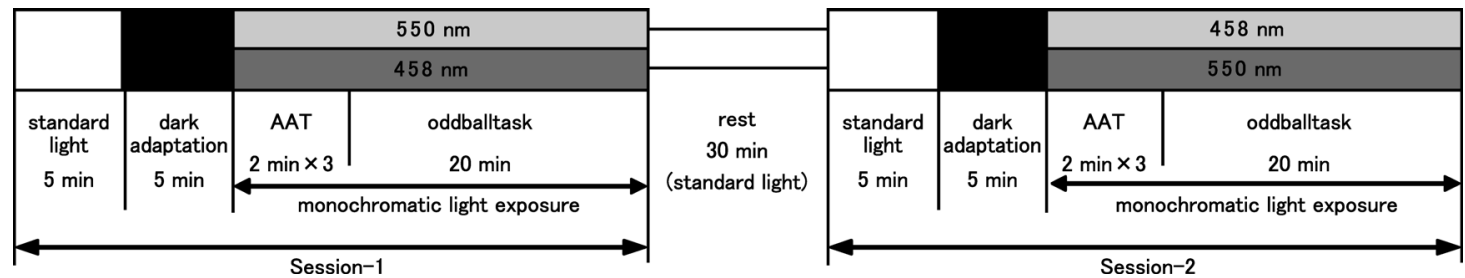

Fig. 1 Overview of the protocol design.

Experimental protocol. After $5 \mathrm{~min}$ of standard light exposure, subjects were dark-adapted for $5 \mathrm{~min}$, followed by 20 min of monochromatic light exposure including a $6 \mathrm{~min}$ AAT and a $20 \mathrm{~min}$ oddball task. The session interval was 30 minutes, while the order of monochromatic light exposure was counterbalanced across subjects. Subjective alertness scores (KSS) were collected before each session.

when measured vertically and $96 \mathrm{~lx}$ when measured horizontally at the eye level), subjects were dark adapted $(<1$ 1x) for $5 \mathrm{~min}$, followed by monochromatic light exposure either at $458 \mathrm{~nm}$ or $550 \mathrm{~nm}$ under the same irradiance density $(9.8$ $\mu \mathrm{W} / \mathrm{cm}^{2}$ at the level of the eye). During monochromatic light exposure, subjects performed an AAT for 6 min to assess the arousal level at the beginning, followed by an approximately 20 min oddball task to extract P300 event-related potentials. The session interval was $30 \mathrm{~min}$, while the order of monochromatic light exposure was counterbalanced by subjects. Subjective alertness scores, as assessed by the Kwansei Gakuin sleepiness scale (KSS; Ishihara et al., 1982), were collected before each session. During the experiment, the subjects kept their eyes open except when they were closed for resting during the AAT.

\section{Monochromatic light exposure}

Monochromatic light was generated by a variable voltage $(0-100)$ halogen lamp, and filtered by interference filters of 10 nm FWHM (FILTER INT 458 NM or 550NM, Co. Edmund Industrial Optics, USA). Light was administered through an acrylic board placed $30 \mathrm{~cm}$ away from the eye. The spectral distribution and the irradiance density of $458 \mathrm{~nm}$ and $550 \mathrm{~nm}$ light were measured by a spectroradiometer (HSR-8100, Maki Manufacturing Co., Ltd.). The target irradiance density at the level of the eye was $9.8 \mu \mathrm{W} / \mathrm{cm}^{2}$ for both $458 \mathrm{~nm}$ and $550 \mathrm{~nm}$ light.

In order to study the time-of-day effects, it is necessary to assess cognitive function over the 24-h cycle. Because the homeostatic drive for sleep usually rises when prior wakefulness exceeds $16 \mathrm{~h}$, and sleep inertia lasts up to $4 \mathrm{~h}$ after awakening, it is plausible that monochromatic light could exert its strongest alerting action during these periods. Furthermore, Lockley et al. (2006) showed that monochromatic light exposure induces robust acute alerting effects at 9.25 hours before the respective averaged wake time during each subject's baseline days. Therefore, in the present study, the subjects underwent monochromatic light exposure two times (daytime and nighttime), $12 \mathrm{~h}$ out of phase with each other. Nighttime light exposure occurred approximately 9.25 hours before the respective averaged wake time observed during each subject's baseline days.

\section{EEG and P300 recordings}

P300 was elicited using an oddball task. The standard (1000 $\mathrm{Hz}$ ) and target stimuli $(2000 \mathrm{~Hz})$ were presented binaurally over earphones (MDR-CD2000, Sony Co.), with a duration of $50 \mathrm{~ms}$ and $10 \mathrm{~ms}$ rise/fall times that were edited using SoundEdit (Macromedia, Inc.) and generated by a computer (iBook M8597J/A, Apple Computer, Inc.). The inter-stimulus interval was varied between $2.0-3.0 \mathrm{~s}$. The target stimuli occurred randomly with a 0.2 probability, and the stimulus intensity was maintained at $70 \mathrm{~dB}$ SPL throughout the experiment. During all sessions, subjects kept their eyes open and, in order to reduce eye movement artifacts in the EEG, were requested to fix their gazes on a small black point on the opal acrylic board and to try not to blink. Subjects were instructed to react as quickly as possible to the target stimulus by pressing a button mounted near the right hand but to do nothing when the standard stimulus was detected.

EEG activity was recorded with $\mathrm{Ag} / \mathrm{AgCl}$ electrodes at the $\mathrm{Fz}, \mathrm{Cz}$, and $\mathrm{Pz}$ electrode sites of the international 10-20 system, with impedances of $10 \mathrm{k} \Omega$ or less. Linked earlobes were used as a reference with a forehead ground. A bipolar electrooculogram (EOG) was recorded with electrodes placed at the outer canthus and below the right eye. The EEG and EOG were amplified by a multichannel bioamplifier (MME3124, Nihon Kohden). The EEG bandpass filter was 0.53-30 $\mathrm{Hz}$; the EOG bandpass filter was $0.016-15 \mathrm{~Hz}$; and the EEG was digitized at $1 \mathrm{~ms} /$ point for $800 \mathrm{~ms}$ with a prestimulus baseline of $100 \mathrm{~ms}$. Waveforms were averaged offline, and trials in which the EEG or EOG exceeded $\pm 75 \mu \mathrm{V}$ were rejected. Response registrations were controlled by a computer (dynabook TX/570LSBB, TOSHIBA). The P300 amplitude was measured relative to the pre-stimulus baseline, and was defined as the largest positive-going peak occurring after the N100-P200-N200 complex, within a latency window between 250 and $550 \mathrm{~ms}$. Reaction time (RT) was defined as the time from the onset of the stimulus until the subject pressed the button, and any RT value longer than $550 \mathrm{~ms}$ or shorter than $150 \mathrm{~ms}$ was rejected. At least 30 artifact-free target presentations were obtained for each session.

\section{Sleepiness and objective assessments of arousal level}

KSS (Ishihara et al., 1982) was used for the assessment of subjective sleepiness. This is a subjective rating scale of 
drowsiness that has been translated into Japanese, and which is based on the SSS (Stanford Sleepiness Scale). The scale values of answers to 22 questions concerning drowsiness were determined. The higher the whole sum, the more drowsy the participant felt.

AAT, which was established by Michimori et al. (1994), was used for the assessment of objective arousal level. In AAT, a cycle including a 1-min period with eyes open followed by a 1min period with eyes closed was repeated three times reciprocally. AAC is calculated by alternating closed-eyes with open-eyes and dividing the $\alpha$-wave power at closed-eyes by the $\alpha$-wave power at open-eyes. AAC rises when the arousal level rises, and falls when the arousal level falls.

\section{Data analysis}

A three-way analysis of variance (ANOVA) test for repeated measurements (time of day $\times$ wavelength $\times$ electrode site) was carried out for P300 latency and amplitude and AAC. Two-way repeated measures ANOVA (time of day $\times$ wavelength) was carried out for RT. A one-way analysis of variance (ANOVA) test for repeated measurements (experimental session) was carried out for the values of subjective assessment of sleepiness measured using the KSS. Paired t-tests were used to compare the differences between $458 \mathrm{~nm}$ light and 550 light conditions, daytime and nighttime condition. For post hoc comparisons, Bonferroni tests were used to compare the differences among the electrode sites. The level of statistical significance for all of these analyses was set at 0.05 .

\section{Results}

\section{Subjective sleepiness}

There were no significant differences in KSS ratings among the experimental sessions $(\mathrm{F}(3,11)=0.717, p>0.05)$.

\section{Alpha attenuation coefficient}

A significant main effect of time of day was noted in AAC $(\mathrm{F}(1,11)=10.89, p<0.01)$, as the $\mathrm{AAC}$ at nighttime was higher than in the daytime (Fig. 2). A significant main effect of electrode site was also noted in AAC $(\mathrm{F}(2,22)=4.967$, $p<0.05)$, as the AAC recorded at the $\mathrm{Pz}$ electrode site was significantly higher than that recorded at the Fz electrode site $(p<0.05)$. No significant main effect of wavelength was found in $\operatorname{AAC}(\mathrm{F}(1,11)=0.265, p>0.05)$.

\section{Event-related potential}

There were no significant main effects in RT for the wavelength $(458 \mathrm{~nm}, 550 \mathrm{~nm})(\mathrm{F}(1,11)=0.003, p>0.05)$, or for the time of day (daytime, nighttime) $(\mathrm{F}(1,11)=0.06, p>0.05)$.

There was a significant interaction between time of day and electrode site in the P300 latency $(\mathrm{F}(2,22)=6.074, p<0.01)$. The P300 latency recorded at Fz was longer than that at the Pz electrode site.

A significant main effect of wavelength was found in P300 amplitude, as exposure to $458 \mathrm{~nm}$ monochromatic light caused

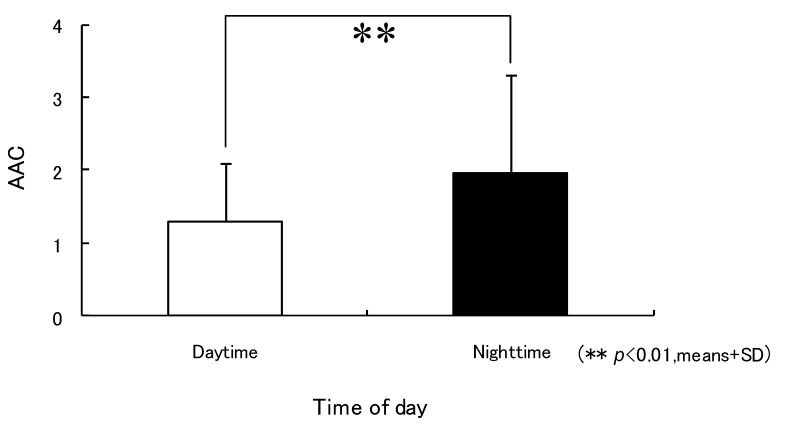

Fig. 2 Globe mean AAC for each time of day. Globe mean AAC for each time of day. A significant main effect of time of day was noted in AAC $(p<0.01)$, as the AAC at nighttime was higher than in the daytime. Data are shown as means + SD.

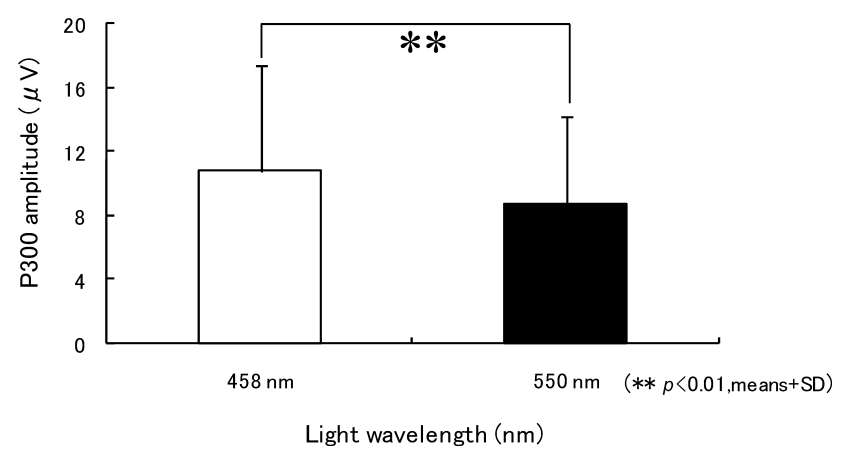

Fig. 3 Globe mean P300 amplitude at $458 \mathrm{~nm}$ and $550 \mathrm{~nm}$. Globe mean P300 amplitude at $458 \mathrm{~nm}$ and $550 \mathrm{~nm}$. A significant main effect of wavelength in the P300 amplitude was observed $(p<0.01)$. The P300 amplitude was significantly larger at $458 \mathrm{~nm}$ than at $550 \mathrm{~nm}(p<0.01)$. Data are shown as means + SD.

a significantly larger P300 amplitude compared with $550 \mathrm{~nm}$ monochromatic light $(\mathrm{F}(1,11)=11.237, p<0.01)$ (Fig. 3). No significant main effect of time of day was found in P300 amplitude $(\mathrm{F}(1,11)=0.001, p>0.05)$. There was a significant interaction among wavelength, time of day, and electrode site $(\mathrm{F}(2,22)=2.742, p<0.05)$. Exposure to $458 \mathrm{~nm}$ monochromatic light induced a larger P300 amplitude at the Fz electrode site at nighttime than in the daytime $(p<0.05)$ (Fig. 4). A significant main effect of electrode site was found in P300 amplitude $(\mathrm{F}(2,22)=39.145, p<0.01)$. P300 amplitude recorded at $\mathrm{Fz}$ was significantly smaller than those recorded at the $\mathrm{Cz}$ and $\mathrm{Pz}$ electrode site $(p<0.01)$.

\section{Discussion}

The present study investigated whether the effects of time of day on cognitive function are modulated by light exposure in a wavelength-dependent manner. Subjective alertness scores, as assessed by KSS (Ishihara et al., 1982), were collected before each session. We found that the subjective sleepiness before monochromatic light exposure was almost at the same level for all of the experimental sessions.

Previous studies showed that AAC rises when the arousal 


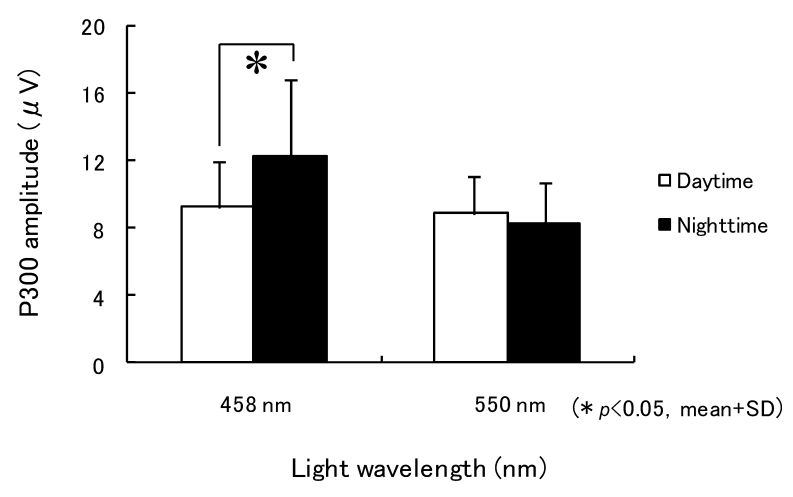

Fig. 4 Mean P300 amplitude at $458 \mathrm{~nm}$ and $550 \mathrm{~nm}$ for each time of day. Mean P300 amplitude at $458 \mathrm{~nm}$ and $550 \mathrm{~nm}$ for each time of day. The P300 amplitude was significantly larger at nighttime than in the daytime during $458 \mathrm{~nm}$ light exposure at the $\mathrm{Fz}$ electrode site $(p<0.05)$. Data are shown as means $+\mathrm{SD}$.

level rises and falls when the arousal level falls (Michimori et al., 1994). In our current study, at the beginning of the monochromatic light exposure, subjects performed an AAT for 6 minutes in order to assess their arousal level. Our results showed that $\mathrm{AAC}$ at nighttime was higher than in the daytime, suggesting that the subject's level of arousal at nighttime was higher than in the daytime when subjects were exposed to 458 $\mathrm{nm}$ or $550 \mathrm{~nm}$ light, although their subjective sleepiness was almost at the same level as before the light exposure.

Using event-related potential-P300, we found that, while subjects performed an oddball task after the Alpha Attenuation Test, a greater $\mathrm{P} 300$ amplitude was elicited following exposure to $458 \mathrm{~nm}$ light than to $550 \mathrm{~nm}$ light, which suggests that 458 $\mathrm{nm}$ light exposure enhanced human higher cognitive function as compared with $550 \mathrm{~nm}$ light exposure. This result is consistent with the findings of Lockley et al. (2006), who found that subjects exposed to $460 \mathrm{~nm}$ light had significantly increased waking EEG power density in the high-alpha range $(9.5-10.5 \mathrm{~Hz})$ as compared with those exposed to $555 \mathrm{~nm}$ light, and extends these findings in terms of higher cognitive function by using an endogenous ERP component, P300. Because the cone system and thus the visual system is not very sensitive to extreme blue and extreme red light, and has its maximum sensitivity for green-yellow light (Bommel, 2006), and the experimental design contrasted two narrow-band monochromatic lights, namely $458 \mathrm{~nm}$ and $550 \mathrm{~nm}$ light with an equal level of irradiance density $\left(9.8 \mu \mathrm{W} / \mathrm{cm}^{2}\right)$, our findings suggest that the melanopsin-dependent photoreception system contributed to the mediation of these responses relative to the visual photopic system. In terms of time-of-day effects on cognitive function, our results showed that exposure to $458 \mathrm{~nm}$ monochromatic light induced a larger P300 amplitude at nighttime than in the daytime at the Fz electrode site, a location that is involved in maintaining an endogenous attentional set in working memory (Ishihara and Yoshii, 1972; Aftanas and Golocheikine, 2001; Kubota et al., 2001; Ishii et al., 1999). Because P300 amplitude is believed to be related to the amount of cognitive resources available for the evaluation of stimuli (Gaillard, 1988), this result suggests that during 458 $\mathrm{nm}$ light exposure, a relatively larger allocation of attention resources had been provided to perform the oddball task at nighttime than in the daytime. The mechanism by which monochromatic light regulates the cognitive function is not fully understood. It is known that intrinsically photosensitive retinal ganglion cells project directly to the ventrolateral preoptic nucleus (VLPO) (Gooley et al., 2003). On the other hand, the VLPO innervates all of the major nuclei of the ascending monoaminergic and, in particular, histaminergic pathways, which are thought to play a key role in wakefulness and EEG arousal (Aston-Jones et al., 1999; Lin et al., 1996). Thus, the effect of $458 \mathrm{~nm}$ light on human cognition might occur indirectly via the VLPO.

We conclude that the time-of-day effects of monochromatic light exposure on cognitive function are modulated in a wavelength-dependent manner. The present study is the first study that focused on the non-visual effects on human cognitive function under the same irradiance density using P300; furthermore, it extends the previous findings in terms of the time-of-day effects. The implications of these findings may be significant for the understanding of the effects of light on human cognition, and may be useful in the design of novel light devices. Further study is needed to explore the time-ofday effects on individual differences.

Acknowledgments This work was supported in part by the Grant-in-Aid for Science Research (B) (grant No. 20370098) from the Japan Society for the Promotion of Science.

\section{References}

Aftanas L, Golocheikine S (2001) Human anterior frontal midline theta and lower alpha reflect emotionally positive state and internalized attention: high-resolution EEG investigation of meditation. Neurosci Lett 310: 57-60

Aston-Jones G, Rajkowski J, Cohen J (1999) Role of locus coeruleus in attention and behavioral exibility. Biol Psychiatry 46: 1309-1320

Badia P, Myers B, Boecker M, Culpepper J, Harsh JR (1991) Bright light effects on body temperature, alertness, EEG and behavior. Physiol Behav 50: 583-588

Berson DM, Dunn FA, Motoharu T (2002) Phototransduction by retinal ganglion cells that set the circadian clock. Science 295: 1070-1073

Boivin DB, Duffy JF, Kronauer RE, Czeisler CA (1996) Doseresponse relationships for resetting of human circadian clock by light. Nature 379: 540-542

Bojkowski CJ, Aldhous ME, English J, Franey C, Poulton AL, Skene DJ, Arendt J (1987) Suppression of nocturnal plasma melatonin and 6-sulphatoxymelatonin by bright and dim light in man. Horm Metab Res 19: 437-440

Bommel WJM (2006) Non-visual biological effect of lighting 
and the practical meaning for lighting for work. Appl Ergon 37: 461-466

Brainard GC, Rollag MD, Hanifin JP (1997) Photic regulation of melatonin in humans: ocular and neural signal transduction. J Biol Rhythms 12: 575-578

Broughton R, Aguirre M, Dunham W (1988) A comparison of multiple and single sleep latency and cerebral evoked potential (P300) measures in the assessment of excessive daytime sleepiness in narcolepsy-cataplexy. Sleep 11: 537-545

Cajochen C, Dijk DJ, Borbély AA (1992) Dynamics of EEG slow-wave activity and core body temperature in human sleep after exposure to bright light. Sleep 15: 337-343

Cajochen C, Zeitzer JM, Czeisler CA, Dijk DJ (2000) Doseresponse relationship for light intensity and ocular and electroencephalographic correlates of human-alertness. Behav Brain Res 115: 75-83

Cajochen C, Munch M, Kobialka S, Krauchi K, Steiner R, Oelhafen P, Orgul S, Wirz-Justice A (2005) High sensitivity of human melatonin, alertness, thermoregulation, and heart rate to short wavelength light. J Clin Endocrinol Metab 90: 1311-1316

Campbell SS, Dawson D (1990) Enhancement of nighttime alertness and performance with bright ambient light. Physiol Behav 48: 317-320

Coull JT (1998) Neural correlates of attention and arousal: insights from electrophysiology, functional neuroimaging and psychopharmacology. Prog Neurobiol 55: 343-361

Deacon S, Arendt J (1995) Melatonin-induced temperature suppression and its acute phase-shifting effects correlate in a dose-dependent manner in humans. Brain Res 688: 77-85

Dijk DJ, Cajochen C, Borbély AA (1991) Effect of a single 3hour exposure to bright light on core body temperature and sleep in humans. Neurosci Lett 121: 59-62

Donchin E (1981) Surprise!...Surprise? Psychophysiology 18: 493-513

Donchin E, Karis D, Bashore TR, Coles MGH, Gratton G (1986) Cognitive psychophysiology and human information processing. In Coles, MGH., Donchin, E and Porges, SW, Eds. Psychophysiology: Systems, Processes and Applications, The Guilford Press, New York, 244-267

Donchin E, Coles GH (1988) Is the P300 component a manifestation of context updating? Behav Brain Sci 11: 357-374

Gaillard AW (1988) Problems and paradigms in ERP research. Biol Psychol 26: 91-109

Gooley JJ, Lu J, Fischer D, Saper CB (2003) A broad role for melanopsin in nonvisual photoreception. J Neurosci 23: 7093-7106

Higuchi S, Liu Y, Yuasa T, Maeda A, Motohashi Y (2000) Circadian variation in the P300 component of human cognitive event-related potential. Chronobiol Int 17: 669-678

Horne JA, Östberg O (1976) A self-assessment questionnaire to determine morningness-eveningness in human circadian rhythms. Int J Chronobiol 4: 97-100

Huang J, Katsuura T, Shimomura Y, Iwanaga K (2006) Diurnal Changes of ERP Response to Sound Stimuli of Varying Frequency in Morning-Type and Evening-Type Subjects. J Physiol Anthropol 25: 49-54

Ishihara K, Saito T, Miyata Y (1982) Sleepiness scale and an experimental approach. Jpn J Psychol 52: 362-365 [In Japanese]

Ishihara T, Yoshii N (1972) Multivariate analytic study of EEG and mental activity in juvenile delinquents. Electroencephalogr Clin Neurophysiol 33: 71-80

Ishii R, Shinosaki K, Ukai S, Inouye T, Ishihara T, Yoshimine T, Hirabuki N, Asada H, Kihara T, Robinson S, Takeda M (1999) Medial prefrontal cortex generates frontal midline theta rhythm. Neuroreport 10: 675-679

Katsuura T, Yasuda T, Shimomura Y, Iwanaga K (2007) Effects of Monochromatic Light on Time Sense for Short Intervals. J Physio Anthropol 26: 95-100

Kleitman N (1939) Sleep and Wakefulness. University of Chicago Press, Chicago (Revised and enlarged edition 1963)

Kok A (1997) Event-related potential (ERP) reflections of mental resources: a review and synthesis. Biol Psychol 45: 19-56

Kubota Y, Sato W, Toichi M, Murai T, Okada T, Hayashi A, Sengoku A (2001) Frontal midline theta rhythm is correlated with cardiac autonomic activities during the performance of an attention demanding meditation procedure. Cogn Brain Res 11: 281-287

Lewy AJ, Wehr TA, Goodwin FK, Newsome DA, Markey SP (1980) Light suppresses melatonin secretion in humans. Science 210: 1267-1269

Lin JS, Hou Y, Sakai K, Jouvet M (1996) Histaminergic descending inputs to the mesopontine tegmentum and their role in the control of cortical activation and wakefulness in the cat. J Neurosci 16: 1523-1537

Lockley SW, Brainard GC, Czeisler CA (2003) High sensitivity of the human circadian melatonin rhythm to resetting by short wavelength light. J Clin Endocrinol Metab 88: 4502-4505

Lockley SW, Evans EE, Scheer FA, Brainard GC, Czeisler CA, Aeschbach D (2006) Short-wavelength sensitivity for the direct effects of light on alertness, vigilance, and the waking electroencephalogram in humans. Sleep 29: 161-168

Michimori A, Stone P, Aguirre A, Stampi C (1994) Analysis of alpha attenuation test. J Sleep Res 23: 454

Myers BL, Badia P (1993) Immediate effects of different light intensities on body temperature and alertness. Physiol Behav 54: 199-202

Pfefferbaum A, Christensen C, Ford JM, Kopell BS (1986) Apparent response incompatibility effects on P300 latency depend on task. Electroencephalogr Clin Neurophysiol 64: 424-437

Polich J, Howard L, Starr A (1983) P300 latency correlates with digit span. Psychophysiology 20: 665-669

Polich J, Kok A (1995) Cognitive and biological determinants 
of P300: an integrative review. Biol Psychol 41: 103-146

Polich J (1996) Meta-analysis of P300 normative aging studies. Psychophysiology 33: 334-353

Polich J (1998) P300 clinical utility and control of variability. J Clin Neurophysiol 15: 14-33

Sato M (2005) The Development of Conceptual Framework in Physiological Anthropology. J Physiol Anthropol Appl Human Sci 24: 289-295

Scheer FA, Van Doornen LJ, Buijs RM (2004) Light and diurnal cycle affect autonomic cardiac balance in human; possible role for the biological clock. Auton Neurosci 110: 44-48

Sutton S, Braren M, Zubin J, John ER (1965) Evoked potential correlates of stimulus uncertainty. Science 150: 1187

Vandewalle G, Gais S, Schabus M, Balteau E, Carrier J, Darsaud A, Sterpenich V, Albouy G, Dijk DJ, Maquet P (2007) Wavelength-Dependent Modulation of Brain Responses to a Working Memory Task by Daytime Light Exposure. Cereb Cortex 17: 2788-2795

Verleger R (1988) Event-related potentials and cognition: a critique of the context updating hypothesis and an alternative interpretation of P3. Behav Brain Sci 11: 343-427

Warman VL, Dijk DJ, Warman GR, Arendt J, Skene DJ (2003) Phase advancing human circadian rhythms with short wavelength light. Neurosci lett 342: 37-40

Wesensten NJ, Badia P, Harsh J (1990) Time of day, repeated testing and interblock interval effects on P300 amplitude. Physiol Behav 47: 653-658

Wever RA, Polasek J, Wildgruber CM (1983) Bright light affects human circadian rhythm. Pflugers Arch 396: 85-87

Zaidi FH, Hull JT, Peirson SN, Wulff K, Aeschbach D, Gooley JJ, Brainard GC, Gregory-Evans K, Rizzo JF, Czeisler CA, Foster RG, Moseley MJ, Lockley SW (2007) ShortWavelength light sensitivity of circadian, pupillary, and visual awareness in humans lacking an outer retina. Curr Biol 17: 2122-2128

Zeitzer JM, Dijk DJ, Kronauer RE, Brown EN, Czeisler CA (2000) Sensitivity of the human circadian pacemaker to nocturnal light: melatonin phase resetting and suppression. $\mathrm{J}$ Physiol 526: 695-702

Received: January 28, 2009

Accepted: August 4, 2009

Correspondence to: Ming An, Humanomics Section, Graduate School of Science and Technology, Chiba University, 1-33

Yayoi-cho, Inage-ku, Chiba 263-8522, Japan

Phone: +81-43-251-1111(4546)

Fax: +81-43-290-3087

e-mail: anming@graduate.chiba-u.jp 